\title{
SLC26A4-linked CEVA haplotype correlates with phenotype in patients with enlargement of the vestibular aqueduct
}

\author{
Janet R. Chao ${ }^{1,2}$, Parna Chattaraj ${ }^{1}$, Tina Munjal ${ }^{1}$, Keiji Honda' ${ }^{1}$ Kelly A. King ${ }^{1}$, Christopher K. Zalewski ${ }^{1}$,
}

Wade W. Chien ${ }^{3}$, Carmen C. Brewer ${ }^{1}$ and Andrew J. Griffith ${ }^{1 *}$ (iD

\begin{abstract}
Background: Recessive mutations of coding regions and splice sites of the SLC26A4 gene cause hearing loss with enlargement of the vestibular aqueduct (EVA). Some patients also have a thyroid iodination defect that can lead to multinodular goiter as part of Pendred syndrome. A haplotype of variants upstream of SLC26A4, called CEVA, acts as a pathogenic recessive allele in trans to mutations affecting the coding regions or splice sites of SLC26A4. Our first hypothesis is that CEVA, acting as a pathogenic recessive allele, is correlated with a less severe phenotype than mutations affecting the coding regions and splice sites of SLC26A4. Our second hypothesis is that CEVA acts as a modifier of the phenotype in patients with EVA caused by mutations affecting the coding regions or splice sites of both alleles of SLC26A4 or EVA caused by other factors.
\end{abstract}

Methods: This was a prospective cohort study of 114 individuals and 202 ears with EVA. To test our first hypothesis, we compared the thyroid and auditory phenotypes of subjects with mutations affecting coding regions of both alleles of SLC26A4 with those of subjects carrying CEVA in trans to mutations affecting the coding regions. To test our second hypothesis, we compared the phenotypes associated with the presence versus absence of CEVA among subjects with no coding region mutations, as well as among subjects with mutations affecting coding regions of both alleles.

Results: Subjects carrying CEVA in trans to a mutation of SLC26A4 have a normal thyroid phenotype and less severe hearing loss in comparison to individuals with mutations affecting coding regions of both alleles of SLC26A4. In subjects with no mutant alleles of SLC26A4, hearing loss was more severe in subjects who carry the CEVA haplotype in comparison to non-carriers. There was no correlation of CEVA with the phenotype of subjects with mutations affecting coding regions of both alleles.

Conclusions: CEVA, acting as a likely pathogenic recessive allele, is associated with a less severe phenotype than alleles with a mutation affecting the coding regions or splice sites of SLC26A4. CEVA may act as a genetic modifier in patients with EVA caused by other factors.

Keywords: Deafness, DFNB4, Haplotype, Hearing, Noncoding, Pendred syndrome, SLC26A4

\footnotetext{
* Correspondence: griffita@nidcd.nih.gov

${ }^{1}$ Otolaryngology Branch, National Institute on Deafness and Other

Communication Disorders, National Institutes of Health, 35A Convent Drive,

Room GF103, NIDCD/NIH, Bethesda, MD, USA

Full list of author information is available at the end of the article
}

(c) The Author(s). 2019 Open Access This article is distributed under the terms of the Creative Commons Attribution 4.0 International License (http://creativecommons.org/licenses/by/4.0/), which permits unrestricted use, distribution, and reproduction in any medium, provided you give appropriate credit to the original author(s) and the source, provide a link to the Creative Commons license, and indicate if changes were made. The Creative Commons Public Domain Dedication waiver (http://creativecommons.org/publicdomain/zero/1.0/) applies to the data made available in this article, unless otherwise stated. 


\section{Background}

Enlarged vestibular aqueduct (EVA) is the most common temporal bone malformation detected in ears with sensorineural hearing loss [1]. Some individuals with EVA have recessive mutations of the coding regions and splice sites of the SLC26A4 gene on chromosome 7q (OMIM 274600) [2]. Mutations of SLC26A4 can cause bilateral EVA and a thyroid iodination defect that can lead to multinodular goiter as part of Pendred syndrome (PS) (OMIM 274600) [3]. The iodination defect can be detected with the perchlorate discharge test [4]. The same pathogenic variants of SLC26A4 can also be associated with nonsyndromic EVA (NSEVA), also referred to as nonsyndromic recessive hearing loss DFNB4 (OMIM 600791) [2, 5].

Until recently, there was no known correlation of specific mutations or variants of SLC26A4 with the presence or absence of the PS thyroid phenotype. Variants originally reported to be specifically associated with NSEVA/DFNB4 [6] are now thought to be coincidental hypomorphic variants [7]. However, it was recently reported that some specific mutations are correlated with hearing loss progression or severity in Korean populations $[8,9]$.

We have reported correlations of the number of mutant alleles of SLC26A4 with the thyroid phenotype $[10,11]$, bi-/unilaterality of EVA [11], severity of hearing loss [12, 13], and recurrence probability of EVA in siblings of Caucasian EVA probands [14]. Pendred syndrome, as defined by an abnormal perchlorate discharge test result, is correlated with biallelic mutations of SLC26A4 (M2) [11]. This correlation is less strong when PS is defined as the presence of multinodular goiter. M2 patients almost always have bilateral EVA, whereas unilateral EVA is almost always associated with one (M1) or zero (M0) mutant alleles of SLC26A4 [11]. Severity of hearing loss is greater in M2 EVA ears than in M0 or M1 EVA ears [12, 13].

We recently reported an SLC26A4-linked haplotype, called Caucasian EVA (CEVA), comprised of 12 variants (10 single-nucleotide substitutions and two singlenucleotide deletions) upstream of SLC26A4 [15]. The frequency of the CEVA haplotype among independent control cohorts, not ascertained for hearing loss, was 28 of 947 chromosomes among Europeans and 11 of 676 chromosomes among Admixed Americans [15]. CEVA was far less frequent among Africans (1 of 1314 chromosomes), East Asians (0 of 1008 chromosomes), and South Asians (1 of 971 chromosomes) [15]. The 12 variants span $613 \mathrm{~kb}$ and five other genes within a region of linkage disequilibrium that extends upstream from the first intron of SLC26A4. Some of the variants are intergenic and some are found within introns of the other genes which include BCAP29, DUS4L, COG5,
HBP1, and PRKAR2B. None of these five genes are known to be associated with EVA or any phenotype consistent with EVA. We showed that a chromosome 7 with CEVA and no mutations of coding regions or splice sites of SLC26A4 acts as a mutant allele with incomplete penetrance in trans to the allele with a mutation affecting the coding regions or splice sites in M1 patients [15]. The prevalence of CEVA was also elevated among Caucasian MO EVA subjects, although it did not seem to be necessary or sufficient for the etiology of EVA in M0 subjects [15].

Based on these findings, we hypothesized that the SLC26A4 allele with the CEVA haplotype is correlated with a less severe phenotype than mutations affecting the coding regions or splice sites of SLC26A4. The aim of our current study was to test this hypothesis by comparison of the phenotypes of M2 subjects without CEVA with those of M1 subjects carrying CEVA in trans to a mutation affecting the coding regions or splice sites. A second aim of our study was to test the hypothesis that CEVA acts as a genetic modifier of the phenotype caused by mutations affecting coding regions or splice sites of both alleles of $S L C 26 A 4$ (M2) or by factors other than SLC26A4 mutations (M0).

\section{Methods \\ Subjects}

This study was approved by the Combined Neurosciences Institutional Review Board (IRB) of the National Institutes of Health (Bethesda, Maryland, USA). Written informed consent was obtained from all adult subjects and parents of minor subjects. Race and ethnicity were classified according to our IRB reporting designations. All Caucasians with EVA on at least one side were included in this study. We originally defined a vestibular aqueduct as enlarged if the diameter $>1.5 \mathrm{~mm} \mathrm{[16],} \mathrm{but}$ subsequently revised our criterion to $>1.0 \mathrm{~mm}$ at the midpoint of the course of the vestibular aqueduct [17]. Subjects were categorized as having either unilateral (U) or bilateral (B) EVA. We excluded subjects without original radiologic images to confirm the presence of EVA. SLC26A4 genotypes and CEVA haplotypes have been previously reported [7, 11, 15, 18, 19]. We excluded three subjects whose haplotype was neither CEVA nor reference [15]. Two previously reported subjects [11] (1159 and 1171) could not be included due to absence of samples. Our cohort included 202 ears with EVA in 114 subjects: 88 subjects with bilateral EVA with 26 subjects with unilateral EVA (Table 1). Thirty-one subjects had homozygous or compound heterozygous pathogenic variants (M2), 14 subjects had one pathogenic variant (M1), and 69 subjects had no pathogenic variants affecting splice sites or coding regions of SLC26A4 (M0). 
Table 1 Study subject demographics

\begin{tabular}{|c|c|c|c|c|c|c|}
\hline $\begin{array}{l}\text { Number of Mutant } \\
\text { Alleles of SLC26A4 }\end{array}$ & $\begin{array}{l}\text { SLC26A4-linked } \\
\text { haplotype }\end{array}$ & $\begin{array}{l}\text { Number of subjects } \\
\text { (male/female) }\end{array}$ & $\begin{array}{l}\text { Average age of } \\
\text { subjects (years) }\end{array}$ & $\begin{array}{l}\text { Number of ears } \\
\text { (male/female) }\end{array}$ & $\begin{array}{l}\text { Number of analyzed } \\
\text { ears }^{\mathrm{a}} \text { (male/female) }\end{array}$ & $\begin{array}{l}\text { Average age of } \\
\text { analyzed ears }{ }^{\text {a }} \text { (years) }\end{array}$ \\
\hline \multirow[t]{3}{*}{2} & $C / C$ & $1(0 / 1)$ & 4.8 & $2(0 / 2)$ & $2(0 / 2)$ & 4.8 \\
\hline & $C / R$ & $3(1 / 2)$ & 10.7 & $6(2 / 4)$ & $6(2 / 4)$ & 10.7 \\
\hline & $R / R$ & $27(11 / 16)$ & 17.4 & $52(21 / 31)$ & $48(20 / 28)$ & 18.4 \\
\hline \multirow[t]{2}{*}{1} & $C / R^{b}$ & $11(6 / 5)$ & 7.8 & $20(10 / 10)$ & $20(10 / 10)$ & 7.5 \\
\hline & $R / R$ & $3(2 / 1)$ & 19.7 & $5(3 / 2)$ & $5(3 / 2)$ & 15.8 \\
\hline \multirow[t]{3}{*}{0} & $C / C$ & $4(2 / 2)$ & 12.4 & $7(4 / 3)$ & $7(4 / 3)$ & 13.0 \\
\hline & $C / R$ & $6(3 / 3)$ & 12.4 & $10(5 / 5)$ & $6(3 / 3)$ & 10.1 \\
\hline & $R / R$ & $59(22 / 37)$ & 11.3 & $100(39 / 61)$ & $94(34 / 60)$ & 12.9 \\
\hline Total & & $114(47 / 67)$ & 12.6 & $202(84 / 118)$ & $188(76 / 112)$ & 13.6 \\
\hline
\end{tabular}

C CEVA, $R$ reference (most common haplotype)

${ }^{a}$ Ears with sufficient audiometric data for analysis

${ }^{b}$ CEVA in trans to mutant allele of SLC26A4

\section{Thyroid phenotype}

Two of 114 subjects were excluded from the thyroid phenotype analysis due to a lack of any testing or reports. Thyroid phenotype was categorized as nonsyndromic (NS), Pendred syndrome (PS), or indeterminate (I). Ultrasonography was used to evaluate thyroid size and texture [10, 11]. Subjects with an elevated perchlorate discharge, regardless of thyroid texture or size, were categorized as PS [10,11]. Subjects were categorized as NS if they had normal thyroid size, normal thyroid texture, and normal perchlorate discharge $(\leq 15 \%)$. Subjects were categorized as I if they had any of the following: (1) no record of a perchlorate discharge test; (2) no record of a thyroid ultrasound test; (3) no record of thyroid serologic tests to rule out concurrent confounding thyroid phenotypes; (4) normal perchlorate discharge, normal thyroid texture, and increased thyroid size; or (5) normal perchlorate discharge and a multinodular, enlarged thyroid. One subject (2085) with normal thyroid texture and size but a borderline perchlorate discharge was categorized as I. Subjects with an I phenotype were excluded from further analysis.

\section{Auditory phenotype}

Severity of hearing loss was classified using a fourfrequency $(0.5 / 1 / 2 / 4-\mathrm{kHz})$ pure-tone air-conduction threshold average (PTA) calculated from the most recent complete audiogram for each ear with EVA $[12,13]$. When there was no response to an air-conducted stimulus, we added $5 \mathrm{~dB}$ to the maximum output of the audiometer (i.e. the "no response" value) and used that value as a proxy for analysis. Ears were excluded if there was not at least one complete pure-tone air-conduction audiogram for the ear with EVA prior to or without a cochlear implant $[12,13]$.

\section{Statistical analyses}

Fisher's exact test was used to investigate associations of sex, EVA laterality (unilateral vs. bilateral), and thyroid phenotype with SLC26A4 genotype-haplotype combination. The following comparisons were made: (1) M2 vs. M1/CEVA (2) M2 vs. M2/CEVA (3) M0 vs. M0/CEVA. The Mann-Whitney test was used to compare pure-tone threshold average (PTA) for ears with different genotypehaplotype combinations. These analyses were performed using GraphPad Prism 7 for Mac OS X (GraphPad Software, La Jolla, CA). Stata (StataCorp LLC, College Station, Texas) was used to perform multivariate linear regression analysis to investigate associations of hearing loss in M2 R/R and M1 C/R EVA patients as a function of genotype status (M1 or M2), CEVA (reference or CEVA) haplotype status, age, sex, and EVA laterality. We performed the same analysis in M0 EVA patients but without genotype status as a variable since all patients were M0.

\section{Results}

To test the hypothesis that CEVA, acting as a recessive mutant allele, is correlated with a less severe phenotype than mutations affecting the coding regions or splice sites of SLC26A4, we compared the phenotypes of M2 subjects without CEVA to those of M1 subjects with CEVA in trans to an allele with the reference haplotype and a mutation affecting the coding regions or splice sites but no CEVA. Among subjects with a determinate thyroid phenotype (Pendred syndrome or nonsyndromic), 10 of 11 M2 subjects without the CEVA haplotype had a Pendred syndrome thyroid phenotype and six of six M1 subjects with CEVA in trans had a nonsyndromic phenotype (Table 2). This difference was significant $(p=0.0006 ; \mathrm{OR}=\infty)$. Twenty-five $(93 \%)$ of $27 \mathrm{M} 2$ subjects without CEVA and nine (82\%) of $11 \mathrm{M} 1$ subjects with CEVA in trans had bilateral EVA. This difference was not significant $(p=0.564)$. The median pure-tone average 
Table 2 Thyroid phenotypes of study subjects

\begin{tabular}{|c|c|c|c|c|}
\hline \multirow[b]{2}{*}{$\begin{array}{l}\text { Number of } \\
\text { Mutant Alleles } \\
\text { of SLC26A4 }\end{array}$} & \multirow[b]{2}{*}{$\begin{array}{l}\text { SLC26A4-linked } \\
\text { haplotype }\end{array}$} & \multicolumn{3}{|c|}{ Number of subjects } \\
\hline & & $\begin{array}{l}\text { Non- } \\
\text { syndromic }\end{array}$ & $\begin{array}{l}\text { Pendred } \\
\text { syndrome }\end{array}$ & Indeterminate \\
\hline \multirow[t]{3}{*}{2} & $\mathrm{C} / \mathrm{C}$ & 0 & 0 & 1 \\
\hline & $\mathrm{C} / \mathrm{R}$ & 0 & 1 & 2 \\
\hline & $R / R$ & 1 & 10 & 16 \\
\hline \multirow[t]{2}{*}{1} & $C / R$ & 6 & 0 & 5 \\
\hline & $\mathrm{R} / \mathrm{R}$ & 1 & 0 & 2 \\
\hline \multirow[t]{3}{*}{0} & $\mathrm{C} / \mathrm{C}$ & 1 & 0 & 3 \\
\hline & $\mathrm{C} / \mathrm{R}$ & 0 & 0 & 6 \\
\hline & $R / R$ & 29 & 0 & 27 \\
\hline Total & & 38 & 11 & 62 \\
\hline
\end{tabular}

NS non-syndromic, PS Pendred syndrome, I indeterminate, C CEVA, $R$ reference

(86.3 dB HL; $n=48$ ears with sufficient audiometric data) in the M2 without CEVA group was significantly different $(p<0.0001 ; 95 \% \mathrm{CI}$ of difference $=-52.5$ to -21.3$)$ from the median pure-tone average $(47.5 \mathrm{~dB} \mathrm{HL} ; n=20$ ears with sufficient audiometric data) in the M1 with CEVA group (Fig. 1). Linear regression analysis determined that among M2 R/R and M1 C/R patients, there is a significant negative correlation between hearing loss and CEVA (patients with CEVA have less severe hearing loss), adjusting for age, sex, and laterality $\left(p<0.001 ; R^{2}=3.94\right)$. These

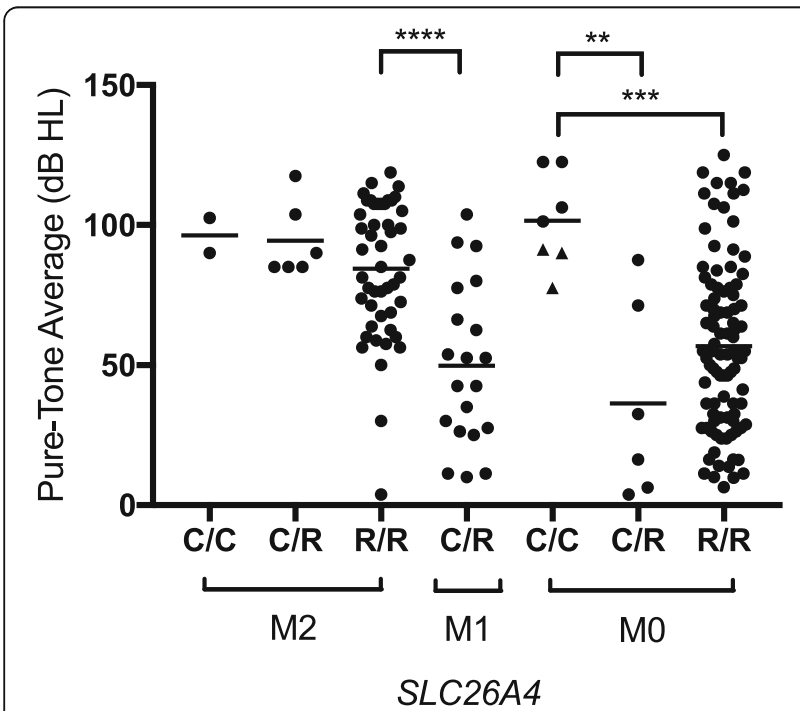

Fig. 1 Four-frequency $(0.5 / 1 / 2 / 4 \mathrm{KHz})$ pure-tone threshold averages for ears with enlargement of the vestibular aqueduct. Each data point represents one ear displayed according to SLC26A4 genotype status (M0, M1 or M2) and haplotype status (C, CEVA; R, reference (most common haplotype)). The CEVA haplotype is trans to the SLC26A4 mutation in the M1 group. ${ }^{* *} p \leq 0.01,{ }^{* * *} p \leq 0.001,{ }^{* * *} p \leq 0.0001$, Mann-Whitney Rank Test. M0 data points shown as a triangle ( $(\boldsymbol{\Delta})$ correspond to CEVA homozygotes with one allele in cis with p.M775 T, a hypofunctional variant thought to be pathogenic only in trans with a mutation affecting the coding region or splice sites results indicate that, as a recessive Mendelian allele in trans to an allele with a mutation of the coding regions or splice sites of SLC26A4, an allele with CEVA but no coding region or splice site mutations is associated with a normal thyroid phenotype and less severe hearing loss in comparison to alleles with a mutation of the coding regions or splice sites and the reference haplotype.

To test the hypothesis that CEVA acts as a modifier of the phenotype of patients with EVA caused by factors other than SLC26A4 mutations, we compared the phenotypes of MO subjects without CEVA with the phenotypes of M0 subjects heterozygous or homozygous for the CEVA haplotype (homozygous SLC26A4 alleles with the CEVA haplotype do not act as pathogenic recessive alleles in M0 subjects [15]). Among M0 subjects with a determinate thyroid phenotype, 31 (100\%) of 31 subjects had a nonsyndromic phenotype irrespective of CEVA haplotype status. Eighteen (31\%) of 59 M0 subjects without CEVA and three (30\%) of $10 \mathrm{M0}$ subjects with CEVA had unilateral EVA. This difference was not significant $(p>0.999)$. The median pure-tone average $(54.4 \mathrm{~dB} \mathrm{HL}$; $n=94$ ears with sufficient audiometric data) in the M0 without CEVA group was significantly different $(p=$ $0.0002 ; 95 \%$ CI of difference $=-1.25$ to 51.3 ) than the median pure-tone average (101.3 dB HL; $n=7$ ears with sufficient audiometric data) in the M0 with homozygous CEVA group (Fig. 1). The latter subjects also had more severe hearing loss $(p=0.0023 ; 95 \% \mathrm{CI}$ of difference $=$ 18.8 to 102.5) than M0 subjects heterozygous for CEVA (24.4 dB HL; $n=6$ ears with sufficient audiometric data) (Fig. 1). Linear regression analysis confirmed this correlation after adjusting for age, sex, and EVA laterality $\left(p=0.003 ; \mathrm{R}^{2}=16.8\right)$. These results indicate that CEVA modifies the severity of hearing loss in M0 EVA ears.

We did not observe any significant differences between hearing loss severity and number of SLC26A4 alleles with the CEVA haplotype within the cohort of M2 subjects $(p=0.3346)$. These results do not support the hypothesis that CEVA modifies the severity of hearing loss caused by mutations affecting the coding regions or splice sites of both alleles of SLC26A4.

\section{Discussion}

We recently reported a haplotype of 12 variants upstream of SLC26A4, termed CEVA, that acts as a pathogenic recessive allele in trans to an allele with a mutation affecting the coding regions or splice sites of SLC26A4 in M1 EVA patients [15]. When American College of Medical Genetics and Genomics criteria are applied to CEVA, there are strong (PS4), moderate (PM5) and supporting (PP1) lines of evidence for its pathogenicity [15], resulting in overall classification as "likely pathogenic." [20]. 
In contrast to individuals with mutations affecting coding regions or splice sites of both alleles of SLC26A4, individuals carrying an SLC26A4 allele with the CEVA haplotype in trans to a mutation of SLC26A4 have a normal thyroid phenotype (i.e. nonsyndromic EVA or DFNB4) and tend to have less severe hearing loss. Although our previous comparisons of phenotypes of M2 subjects with those of M1 subjects revealed that the M1 phenotypes were less severe than the M2 phenotypes, some of the M1 subjects had the reference haplotype in trans to the mutant allele, and some of the M2 subjects had CEVA in cis with one or both mutant alleles. Our current result and conclusion are novel because we performed a comparison that was not confounded by inclusion of M1/reference or M2/CEVA subjects.

Our analysis of M0 subjects suggests that CEVA, acting as a genetic modifier, increases the severity of hearing loss associated with EVA caused by factors other than mutant alleles of SLC26A4. Alternatively, our observation may result from ascertainment bias or other unknown factors among the M0 subjects. In contrast, we did not observe any correlations between hearing loss severity and number of SLC26A4 alleles with the CEVA haplotype within the cohort of M2 subjects. However, the power of these analyses was limited by the number of M2 subjects with CEVA (four). We did not perform this analysis in M1 subjects since CEVA is etiologic in those subjects and cannot also be considered a modifier. Furthermore, we cannot be certain of the etiology of EVA in M1 subjects without CEVA. We could therefore not test the hypothesis that CEVA acts as a genetic modifier in M1 subjects.

It is possible there are pathogenic variants in regions or genes unlinked to SLC26A4 that cause hearing loss and nonsyndromic EVA or modify the severity of hearing loss in ears with EVA. However, the co-segregation of EVA with SLC26A4-linked markers in M1 families, and the near-zero probability of EVA in the siblings of M0 EVA probands, indicate that such variants would be extremely rare Mendelian causes of nonsyndromic EVA [14]. Our results do not address whether variants in unlinked regions and genes modify the hearing loss phenotype in ears with EVA.

We previously recommended inclusion of testing for CEVA along with analysis of SLC26A4 exons and splice sites for individuals with hearing loss and EVA because the detection of CEVA provides a definitive diagnosis for individuals with a mutation affecting the trans allele of SLC26A4 [15]. Our current study directly demonstrates that individuals with this SLC26A4 genotype-haplotype result are unlikely to develop the thyroid abnormality and increased severity of hearing loss associated with Pendred syndrome and mutations affecting the splice sites or coding regions of both alleles of SLC26A4.
Finally, this study indicates that CEVA may act as a genetic modifier to increase the severity of hearing loss in ears with EVA caused by factors other than mutations affecting the coding regions or splice sites of SLC26A4.

\section{Conclusions}

CEVA, acting as a likely pathogenic recessive allele of $S L C 26 A 4$, is associated with less severe auditory and thyroid phenotypes than alleles with a mutation affecting the coding regions or splice sites of SLC26A4. CEVA may act as a genetic modifier in patients with EVA caused by other factors.

\section{Abbreviations \\ CEVA: SLC26A4-linked haplotype associated with enlargement of the vestibular aqueduct; EVA: Enlargement of the vestibular aqueduct; I: Indeterminate thyroid phenotype; M0: Harboring no mutations affecting coding regions or splice sites of SLC26A4; M1: Harboring a mutation affecting coding regions or splice sites of one allele of SLC26A4; M2: Harboring mutations affecting coding regions or splice sites of both alleles SLC26A4; NSEVA: Nonsyndromic enlargement of the vestibular aqueduct; PS: Pendred syndrome; PTA: Pure-tone (hearing) threshold average}

\section{Acknowledgements}

We thank the research subjects for their participation, NIH otolaryngology/ audiology clinic staff, Anne Madeo, Julie Muskett, Anna Clements, Jessica Ratay, H. Jeffrey Kim, John Butman, Thomas Shawker and James Reynolds for clinical support, H. Jeffrey Kim and Thomas Friedman for helpful discussions and critical review of the manuscript, and Michael Setzer for assistance in classifying the pathogenic potential of the CEVA haplotype according to ACMG criteria.

\section{Authors' contributions}

JRC, WWC, CCB and AJG designed the study and interpreted data. JRC, PC, $\mathrm{TM}, \mathrm{KH}, \mathrm{KAK}, \mathrm{CKZ}, \mathrm{WWC}$ and CCB contributed to the acquisition and analysis of data. JRC and AJG drafted the manuscript. AJG conceived and takes overall responsibility for the study. All authors contributed to revisions of the manuscript, and all authors read and approved the final manuscript.

\section{Funding}

This study was supported by NIH intramural research funds Z01-DC-000060 and Z01-DC-000064

\section{Availability of data and materials}

The data that support the findings of this study are available from the corresponding author upon reasonable request.

\section{Ethics approval and consent to participate}

This study was approved by the Combined Neurosciences Institutional Review Board (IRB) of the National Institutes of Health (Bethesda, Maryland, USA). Written informed consent was obtained from all adult subjects and parents of minor subjects.

\section{Consent for publication}

Not applicable.

\section{Competing interests}

The authors declare that they have no competing interests.

\section{Author details}

1Otolaryngology Branch, National Institute on Deafness and Other Communication Disorders, National Institutes of Health, 35A Convent Drive, Room GF103, NIDCD/NIH, Bethesda, MD, USA. 'Division of Otolaryngology, Department of Surgery, Yale School of Medicine, New Haven, CT, USA. ${ }^{3}$ Neurotology Program, National Institute on Deafness and Other Communication Disorders, National Institutes of Health, Bethesda, MD, USA. 
Received: 30 November 2018 Accepted: 24 June 2019

Published online: 02 July 2019

\section{References}

1. Valvassori GE, Clemis JD. Abnormal vestibular aqueduct in cochleovestibular disorders. Adv Otorhinolaryngol. 1978;24:100-5.

2. Usami S, Abe S, Weston MD, Shinkawa H, Van Camp G, Kimberling WJ. Nonsyndromic hearing loss associated with enlarged vestibular aqueduct is caused by PDS mutations. Hum Genet. 1999;104(2):188-92.

3. Everett LA, Glaser B, Beck JC, et al. Pendred syndrome is caused by mutations in a putative sulphate transporter gene (PDS). Nat Genet. 1997; 17(4):411-22.

4. Morgans ME, Trotter WR. Association of congenital deafness with goitre; the nature of the thyroid defect. Lancet. 1958;1 (7021):607-9.

5. Li XC, Everett LA, Lalwani AK, et al. A mutation in PDS causes nonsyndromic recessive deafness. Nat Genet. 1998;18(3):215-7.

6. Scott DA, Wang R, Kreman TM, et al. Functional differences of the PDS gene product are associated with phenotypic variation in patients with Pendred syndrome and non-syndromic hearing loss (DFNB4). Hum Mol Genet. 2000; 9(11):1709-15.

7. Choi BY, Stewart AK, Madeo AC, et al. Hypo-functional SLC26A4 variants associated with nonsyndromic hearing loss and enlargement of the vestibular aqueduct: genotype-phenotype correlation or coincidental polymorphisms? Hum Mutat. 2009;30(4):599-608.

8. Lee HJ, Jung J, Shin JW, et al. Correlation between genotype and phenotype in patients with bi-allelic SLC26A4 mutations. Clin Genet. 2014; 86(3):270-5.

9. Rah YC, Kim AR, Koo JW, Lee JH, Oh SH, Choi BY. Audiologic presentation of enlargement of the vestibular aqueduct according to the SLC26A4 genotypes. Laryngoscope. 2015;125(6):E216-22.

10. Madeo AC, Manichaikul A, Reynolds JC, et al. Evaluation of the thyroid in patients with hearing loss and enlarged vestibular aqueducts. Arch Otolaryngol Head Neck Surg. 2009;135(7):670-6.

11. Pryor SP, Madeo AC, Reynolds JC, et al. SLC26A4/PDS genotype-phenotype correlation in hearing loss with enlargement of the vestibular aqueduct (EVA): evidence that Pendred syndrome and non-syndromic EVA are distinct clinical and genetic entities. J Med Genet. 2005;42(2):159-65.

12. King KA, Choi BY, Zalewski C, et al. SLC26A4 genotype, but not cochlear radiologic structure, is correlated with hearing loss in ears with an enlarged vestibular aqueduct. Laryngoscope. 2010;120(2):384-9.

13. Rose J, Muskett JA, King KA, et al. Hearing loss associated with enlarged vestibular aqueduct and zero or one mutant allele of SLC26A4. Laryngoscope. 2017;127(7):E238-E243. https://doi.org/10.1002/lary.26418. Epub 2016 Nov 15

14. Choi BY, Madeo AC, King KA, et al. Segregation of enlarged vestibular aqueducts in families with non-diagnostic SLC26A4 genotypes. J Med Genet. 2009;46(12):856-61.

15. Chattaraj P, Munjal T, Honda K, et al. A common SLC26A4-linked haplotype underlying non-syndromic hearing loss with enlargement of the vestibular aqueduct. J Med Genet. 2017:54(10):665-73.

16. Valvassori GE, Clemis JD. The large vestibular aqueduct syndrome. Laryngoscope. 1978;88(5):723-8.

17. Boston M, Halsted M, Meinzen-Derr J, et al. The large vestibular aqueduct: a new definition based on audiologic and computed tomography correlation. Otolaryngol Head Neck Surg. 2007;136(6):972-7.

18. Chattaraj P, Reimold FR, Muskett JA, et al. Use of SLC26A4 mutation testing for unilateral enlargement of the vestibular aqueduct. JAMA OtolaryngolHead Neck Surg. 2013;139(9):907-13.

19. Muskett JA, Chattaraj P, Heneghan JF, et al. Atypical patterns of segregation of familial enlargement of the vestibular aqueduct. Laryngoscope. 2016; 126(7):E240-7.

20. Richards S, Aziz N, Bale S, et al. Standards and guidelines for the interpretation of sequence variants: a joint consensus recommendation of the American College of Medical Genetics and Genomics and the Association for Molecular Pathology. Genet Med. 2015;17(5):405-24.

\section{Publisher's Note}

Springer Nature remains neutral with regard to jurisdictional claims in published maps and institutional affiliations.

\section{Ready to submit your research? Choose BMC and benefit from:}

- fast, convenient online submission

- thorough peer review by experienced researchers in your field

- rapid publication on acceptance

- support for research data, including large and complex data types

- gold Open Access which fosters wider collaboration and increased citations

- maximum visibility for your research: over $100 \mathrm{M}$ website views per year

At BMC, research is always in progress.

Learn more biomedcentral.com/submissions 